\title{
Holistic discretisation of shear dispersion in a two-dimensional channel
}

\author{
T. MacKenzie* A. J. Roberts ${ }^{\dagger}$
}

(14 January 2003, corrected August 9, 2018)

\begin{abstract}
Consider the spread of a contaminant along a 2D channel or river. We directly derive the $1 \mathrm{D}$ discrete numerical model from the $2 \mathrm{D}$ advective and diffusive dynamics for the evolution of the contaminant. The holistic discretisation of the $2 \mathrm{D}$ advection-diffusion equation is placed within the purview of centre manifold theory by dividing the physical domain into rectangular $2 \mathrm{D}$ elements through introducing artificial insulating boundaries which are later removed. The resulting holistic discretisation is consistent with the 1D Taylor model for shear dispersion in the channel. This new technique accurately models the subgrid scale processes and provides a
\end{abstract}

${ }^{*}$ Dept. Maths \& Computing, University of Southern Queensland, Toowoomba, Queensland 4352, Australia.

$\dagger$ Dept. Maths \& Computing, University of Southern Queensland, Toowoomba, Queensland 4352, Australia.

mailto: anthony.roberts@adelaide.edu.au

${ }^{0}$ See http://anziamj.austms.org.au/V44/CTAC2001/Mack for this article, (C) Austral. Mathematical Soc. 2018. Published 1 April 2003, amended August 9, 2018. ISSN 1446-8735 
direct link between the 1D numerical discretisation and the original 2D physical dynamics. Centre manifold theory also systematically incorporates the physical inlet and outlet conditions into the 1D discretisation. This method is straightforwardly extended to nonlinear reaction-diffusion equations and more complex geometries.

\section{Contents}

1 Introduction

2 Divide the domain into elements

3 Centre manifold theory underpins the analysis

4 Shear dispersion appears with a low order approximation

5 Inlet and outlet boundary conditions are easily incorporated

C524

6 Conclusion

C527

References

C529

\section{Introduction}

Consider the dispersion of a pollutant or other tracer along a river, channel or pipe. Because of the long narrow geometry we are primarily interested in the evolution along the river, channel or pipe. 


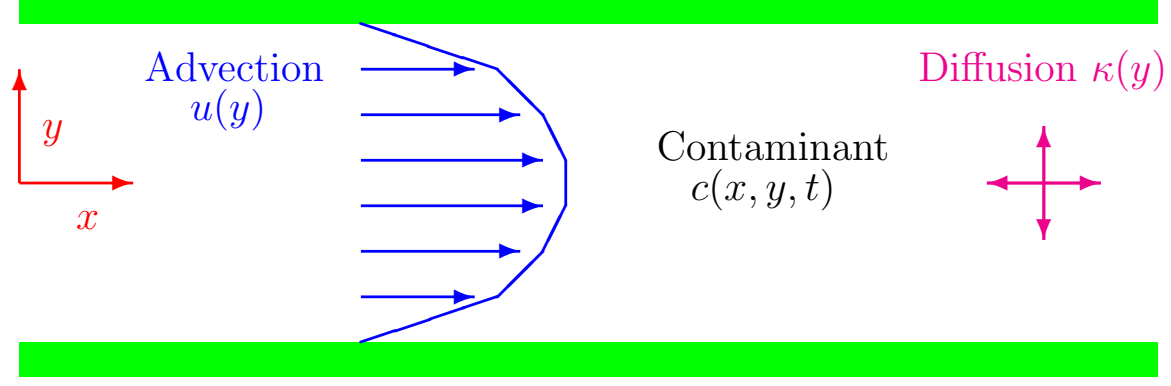

Figure 1: The physical processes of advection and diffusion spread a contaminant along a channel.

Thus instead of solving a three-dimensional advection-diffusion equation we typically seek a one-dimensional model describing the longitudinal dynamics. Then numerical solutions are used for predictions. For definiteness we consider here the advection-diffusion of a contaminant of concentration $c(x, y, t)$ in a two-dimensional channel of non-dimensional width $2 b$ as shown in Figure 1 . The governing advection-diffusion equation is

$$
\frac{\partial c}{\partial t}+u \frac{\partial c}{\partial x}=\boldsymbol{\nabla} \cdot(\kappa \boldsymbol{\nabla} c) \quad \text { in }-b<y<b,
$$

where $x$ measures distance along the channel, $u(y)$ is the laterally varying, longitudinal advection velocity, and $\kappa$ is the coefficient of diffusion. Originally Taylor [13] derived the corresponding onedimensional model,

$$
\frac{\partial C}{\partial t}=-U \frac{\partial C}{\partial x}+D \frac{\partial^{2} C}{\partial x^{2}}
$$

for the cross-channel averaged concentration $C(x, t)$ where $U$ is the cross-channel mean advection velocity and $D \propto U^{2} b^{2} / \kappa$ is an effective dispersion coefficient along the channel. Observe that the dispersion coefficient $D$ is large when the molecular diffusivity $\kappa$ is 
small. The Taylor model (2) would then be discretised on some numerical grid, to say

$$
\frac{\partial C_{j}}{\partial t} \approx-\frac{U}{h}\left(C_{j+1}-C_{j-1}\right)+\frac{D}{h^{2}}\left(C_{j+1}-2 C_{j}+C_{j-1}\right),
$$

in order to make predictions in any given situation. However, there are difficulties in deducing the correct boundary conditions to use at the upstream inlet and the downstream outlet [12]. Smith [12] and Roberts [6] developed arguments for deriving the appropriate boundary conditions for the Taylor model, but the required techniques are difficult even for this simple problem. Instead here we explore bypassing the one-dimensional Taylor model (2) by instead deriving a numerical model, such as (3) or more accurately (15), directly from the physical dynamics expressed in the two-dimensional advection-diffusion problem (1). The analysis is a variant of holistic discretisation introduced in [8]. The application of this technique is explored here in a relatively simple dispersion problem.

There are several benefits of this single step approach of deriving the 1D discretisation (3) directly from the 2D continuum model (1). Firstly, it is simpler; there is a direct connection between the numerical model we obtain in $\S 4$ and the original advection-diffusion equation. Secondly, the derivation described in $\S 5$ of a numerical discretisation near the inlet and outlet is straightforward (as introduced for Burgers' equation in [9]). Lastly, being based upon centre manifold theory [1, 2, e.g.], as argued in $\S 2-3$ we are reasonably assured of fidelity between the numerical model and the original equations. Indeed it is this assurance of fidelity that ensures we derive the appropriate shear dispersion coefficient $D \propto U^{2} b^{2} / \kappa$ in the numerical model (3), without ever assuming large $x$ scales, an assumption formerly absolutely crucial in deriving Taylor's model (2).

To make the problem even more definite we follow Watt and Roberts [14] by deciding that the cross-channel profile of the advec- 
tion velocity and the coefficient of diffusion are both parabolic:

$$
u=U \frac{3}{2}\left(1-y^{2} / b^{2}\right), \quad \text { and } \quad \kappa=\mathcal{K}\left(1-y^{2} / b^{2}\right) .
$$

The advantage of these particular choices is that the subgrid scale field is conveniently found analytically - other choices require numerical solutions in deriving the numerical model. These choices are physically relevant to dispersion in a river, estuary or channel where turbulent mixing varies across the channel due to, for example, cross-channel variations in depth. Partnering this choice for the (turbulent) diffusivity $\kappa(y)$ are boundary conditions of zero flux of contaminant $c$ across the sides of the channel: because $\kappa \rightarrow 0$ as $y \rightarrow \pm b$ a sufficient condition is

$$
\frac{\partial c}{\partial y} \text { is bounded on } y= \pm b .
$$

We non-dimensionalise with respect to the channel half-width, $b$, and a cross channel diffusion time, $b^{2} / \mathcal{K}$ : in effect $b=\mathcal{K}=1$ and $U$ is replaced by the Peclet number $\mathcal{P}=U b / \mathcal{K}$. See that $\mathcal{P} b$ is the downstream advection distance in a cross channel diffusion time. In application the Peclet number is typically large.

These specific choices form a problem which was studied by Watt and Roberts [14]. They derived the one-dimensional Taylor model, generalised to higher order,

$$
\begin{aligned}
\frac{\partial C}{\partial t}= & -\mathcal{P} \frac{\partial C}{\partial x}+\left(\frac{2}{3}+\frac{\mathcal{P}^{2}}{30}\right) \frac{\partial^{2} C}{\partial x^{2}}+\left(-\frac{2 \mathcal{P}}{45}+\frac{\mathcal{P}^{3}}{630}\right) \frac{\partial^{3} C}{\partial x^{3}} \\
& +\left(\frac{2}{135}-\frac{\mathcal{P}^{2}}{315}-\frac{13 \mathcal{P}^{4}}{189000}\right) \frac{\partial^{4} C}{\partial x^{4}}+\cdots
\end{aligned}
$$

where $C(x, t)$ represents any reasonable measure of the contaminant at station $x$ such as the mid-channel value which we use herein. This generalised Taylor model reappears in the equivalent PDE (18) to the discretisation (15) derived in $§ 4$. 

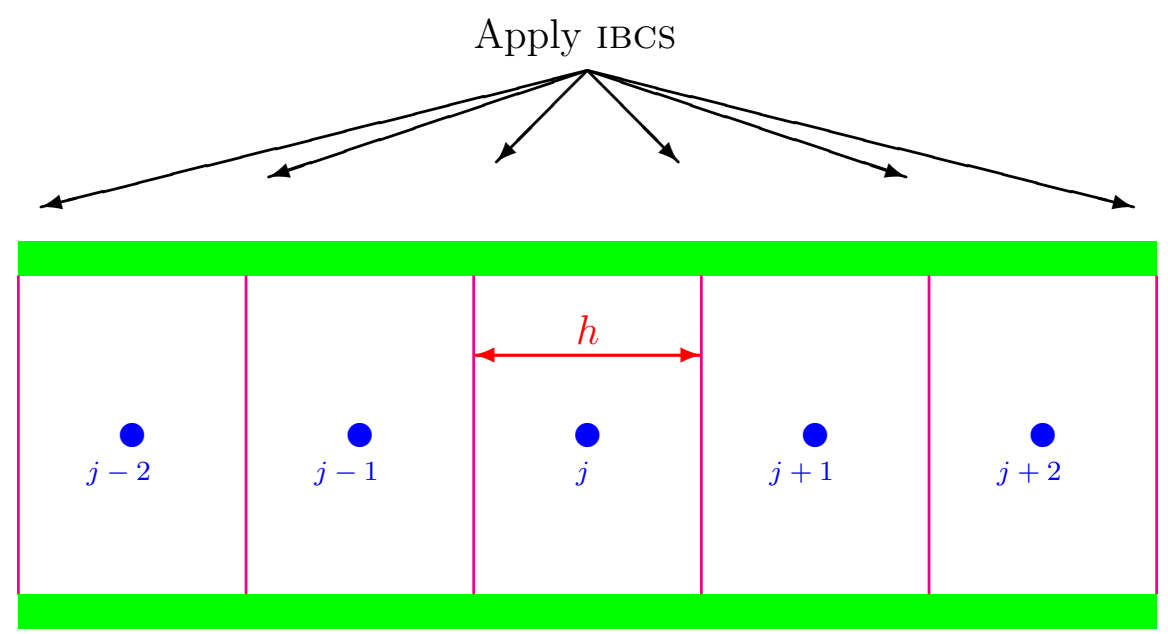

FiguRE 2: The discretisation of the 2D channel into rectangular elements. The $j$ th element is centred upon the grid point $x_{j}$.

\section{Divide the domain into elements}

We divide the physical domain into $m$ elements. Artificial boundaries are applied between elements to isolate each from its neighbours and centre manifold theory is applied as discussed in $\S 3$. Rosencrans [11] analogously divided a periodically varying channel into elements in order to use multiple scales to derive a Taylor model for the large scale dispersion. Here, the isolating internal boundaries are later removed in the analysis to form the relevant discretisation.

Here we divide domain into rectangular elements of longitudinal size $h$ and extending across the whole channel, $-1<y<1$ as illustrated in Figure 2. We place grid points, $x_{j}=j h$ and $y_{j}=0$, at the centre of these elements. Other shaped elements are also possible, but we only consider rectangular elements herein. We then apply the following internal boundary conditions (IBCS) to each of 
the elements

$$
\begin{aligned}
& c_{j}\left(x_{j+1}, y, t\right)=(1-\gamma) c_{j}\left(x_{j}, y, t\right)+\gamma c_{j+1}\left(x_{j+1}, y, t\right), \\
& c_{j}\left(x_{j-1}, y, t\right)=(1-\gamma) c_{j}\left(x_{j}, y, t\right)+\gamma c_{j-1}\left(x_{j-1}, y, t\right),
\end{aligned}
$$

except for the elements immediately adjacent to the physical extreme upstream and downstream boundaries at the inlet and the outlet as discussed in $\S 4$. In 1D these IBCS ensure consistency [10] and here are straightforwardly extended to 2D. Important features of the internal boundary conditions are that: when evaluated at $\gamma=0$ the elements are effectively isolated from each other, providing the basis for the application of centre manifold theory; whereas when evaluated at $\gamma=1$ the continuity in the concentration field between elements is effectively restored and the resulting discretisation models the original advection-diffusion dynamics throughout the channel.

The parameter $\gamma$ is an interelement coupling parameter. It controls the flow of information between adjacent elements: if only $\gamma$ terms are retained in the asymptotic expansions then each element is coupled with the adjacent element on the left and right; if $\gamma^{2}$ terms are retained then each element is coupled with the nearest two elements on the left and right; and so on.

\section{Centre manifold theory underpins the analysis}

Centre manifold theory provides sound theoretical support for the discretisation. We develop the application of the theory from that introduced in [8] for the one dimensional Burgers' equation and extended to two dimensions in [4]. 
The approach is based upon a linear picture of the dynamics. We begin by the mathematical trick of formally adjoining the dynamically trivial equations

$$
\frac{\partial \gamma}{\partial t}=\frac{\partial \mathcal{P}}{\partial t}=0
$$

to the advection-diffusion equation (1), side boundary condition (5) and the internal boundary condition (7-8). The introduction of (9) allows us to treat all terms multiplied by $\gamma$ or $\mathcal{P}$ as "nonlinear" pertubations. With the isolating boundaries, (7-8) with $\gamma=0$, around each element the "linear" dynamics, namely the diffusion equation

$$
\frac{\partial c}{\partial t}=\nabla \cdot\left(\left(1-y^{2}\right) \nabla c\right) \quad \text { such that } \quad c\left(x_{j \pm 1}, y, t\right)=c\left(x_{j}, y, t\right),
$$

as $\mathcal{P}=0$, result in the concentration of contaminant in each element evolving exponentially quickly to a constant value in each element. This is a set of fixed points in the extended state space $(c(x, y), \gamma, \mathcal{P})$. We then use series expansions in the nonlinear parameters $\gamma$ and $\mathcal{P}$ to perturb this fixed point to account for coupling between the elements (non-zero $\gamma$ ) and downstream advection (non-zero $\mathcal{P}$ ). By theory [2, p281], since (10) has $m$ zero eigenvalues and (9) has two, there exists an $m+2$ dimensional centre manifold.

The centre manifold is parametrized by $\gamma, \mathcal{P}$ and a measure of $c$ in each element - we choose the grid value

$$
c_{j}=c\left(x_{j}, 0, t\right) .
$$

This centre manifold $\mathcal{M}$ and the evolution thereon is

$$
c(x, y, t)=v(x, y ; \mathbf{c}, \gamma, \mathcal{P}) \quad \text { such that } \quad \dot{c}_{j}=g_{j}(\mathbf{c}, \gamma, \mathcal{P}),
$$

where $\mathbf{c}$ denotes the vector of $m$ grid values $c_{j}$. The evolution equation (12), evaluated at $\gamma=1$, gives a discrete model for the advection-diffusion in the channel. 
The advantage of using modern dynamical systems theory to develop such finite difference approximations is the sound theoretical support for the method. As well as existence, centre manifold theory assures:

- the relevance of the $m+2$ dimensional dynamics, through exponentially quick decay to $\mathcal{M}$ [2], as an accurate and stable model of the original advection-diffusion dynamics at finite grid size $h$;

- and that we may approximate the shape of the centre manifold and the evolution thereon by approximately solving the associated PDE (13) obtained by substituting (12) into (1) [2].

While centre manifold theory guarantees useful properties in the neighbourhood of the fixed points in $(c(x, y), \gamma, \mathcal{P})$ space, we must evaluate the model (12) at $\gamma=1$. However, the good properties that have been observed in applications of this technique $[10,8,9,3$, 4] such as consistency, increased accuracy and stability for certain regions of the nonlinear parameters, suggest that $\gamma=1$ is within the useful neighbourhood of the origin. Further, in this application the Taylor model (2) for the shear dispersion appears in our numerical model.

\section{Shear dispersion appears with a low order approximation}

To develop the centre manifold model for the contaminant dispersion in a channel we substitute (12) into the advection-diffusion 
equation (1) and solve the following resultant PDE

$$
\frac{\partial c}{\partial t}=\sum_{j} \frac{\partial v}{\partial c_{j}} g_{j}=\nabla \cdot\left(\left(1-y^{2}\right) \nabla v\right)-\mathcal{P} \frac{3}{2}\left(1-y^{2}\right) \frac{\partial v}{\partial x} .
$$

We use computer algebra to handle the algebraic details of constructing the manifold and evolution on the manifold. The basic algorithm introduced by Roberts in $[7,8]$ iterates to drive the residuals of the governing equation (1), the side channel condition (5) and the boundary conditions $(7-8)$ to zero. ${ }^{1}$ In each iteration, we solve a problem of the form

$$
\boldsymbol{\nabla} \cdot\left(\left(1-y^{2}\right) \boldsymbol{\nabla} v^{\prime}\right)=g^{\prime}+\text { residual }
$$

for updates $g^{\prime}$ and $v^{\prime}$ to the subgrid scale structure of the concentration. Generally these subgrid problems have to be solved numerically, but for this particular choice of $\kappa(y)$ and $u(y)$ these are all done algebraically.

We visualize the centre manifold in an element by ploting the field for specific values of $c_{j}$. Figure 3 shows an example of the field $c$ in the channel with $c_{0}=1$ and $c_{j}=0$ for $j \neq 0$. The field models the downstream advection into neighbouring elements and the interaction with diffusion. The subgrid scale field constructed by this holistic technique is comprised of actual solutions to the advection-diffusion dynamics while also accounting for the evolution of the grid values.

Executing the computer algebra to construct a discretisation and retaining up to quadratic terms in the coupling $\gamma$ and the Peclet number $\mathcal{P}$, the numerical model for the shear dispersion in a channel

${ }^{1}$ The REDUCE computer algebra code is available from the authors upon request. 


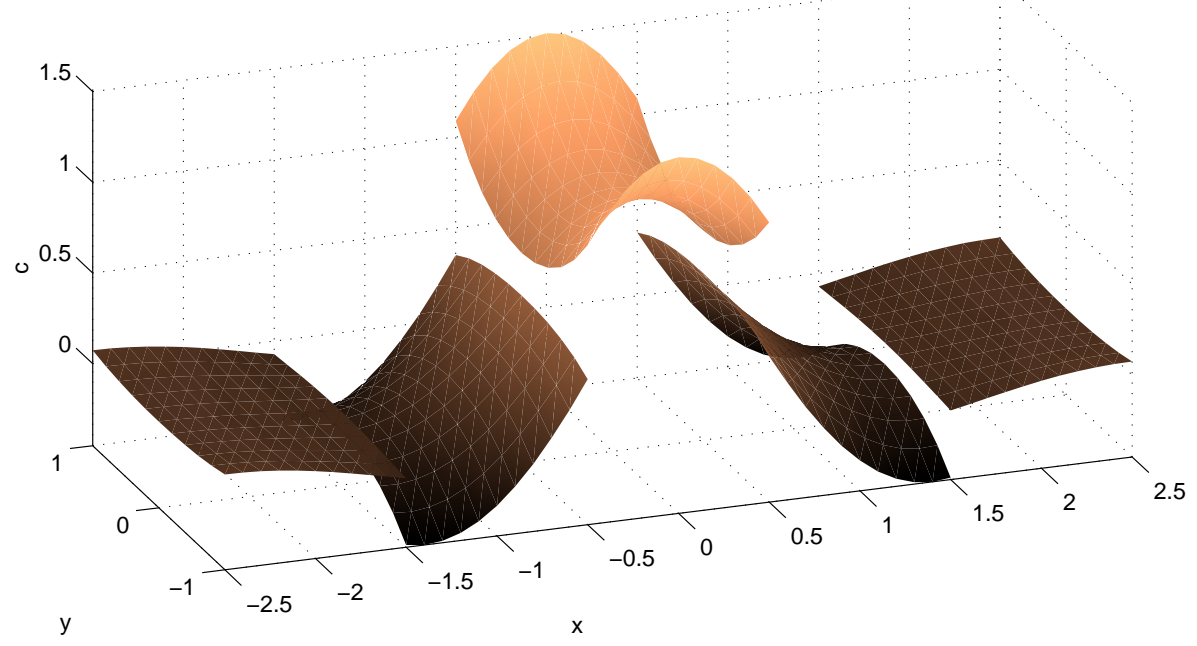

Figure 3: Plot of the two-dimensional field $c(x, y)$ from $\mathcal{M}$ with errors $\mathcal{O}\left(\gamma^{3}, \mathcal{P}^{3}\right)$, in the $j$ th element when $c_{0}=1, c_{ \pm 1}=c_{ \pm 2}=0$, and $\gamma=\mathcal{P}=1$.

is

$$
\begin{aligned}
\frac{\partial c_{j}}{\partial t}= & \frac{2}{3} h^{-2}\left(\gamma \delta^{2}-\frac{\gamma^{2}}{12} \delta^{4}\right) c_{j}+\left(\gamma-\gamma^{2}\right) \frac{\mathcal{P}^{2}}{8} \delta^{2} c_{j}+\gamma^{2} \frac{\mathcal{P}^{2}}{30} h^{-2} \delta^{2} c_{j} \\
& -\mathcal{P} h^{-1}\left(\gamma \mu \delta-\frac{\gamma^{2}}{6} \mu \delta^{3}\right) c_{j}-\gamma^{2} \frac{2 \mathcal{P}}{45} h^{-3} \mu \delta^{3} c_{j} \\
& +\gamma^{2}\left(\frac{2}{135}+\frac{\mathcal{P}^{2} h^{2}}{72}-\frac{\mathcal{P}^{2} h^{4}}{20}\right) h^{-4} \delta^{4} c_{j}+\mathcal{O}\left(\mathcal{P}^{3}, \gamma^{3}\right) \cdot(15)
\end{aligned}
$$

This model is written in terms of centred difference and mean operators, $\delta c_{j}=c_{j+1 / 2}-c_{j-1 / 2}$ and $\mu c_{j}=\left(c_{j+1 / 2}+c_{j-1 / 2}\right) / 2$ respectively. To discuss the discretisation relevant to the original PDE we evaluate the numerical model at $\gamma=1$ - observe in (15) that

- the first term on the right-hand side is an $\mathcal{O}\left(h^{4}\right)$ estimate of 
the mean longitudinal molecular diffusion

$$
\bar{\kappa} \frac{\partial^{2} c}{\partial x^{2}}
$$

- the second term, if truncated to errors $\mathcal{O}\left(\gamma^{2}\right)$, would stablise the discretisation for large advection velocities $\mathcal{P}^{2}$, but here disappears when truncated to errors $\mathcal{O}\left(\gamma^{3}\right)$;

- the third term gives the shear dispersion term

$$
D \frac{\partial^{2} c}{\partial x^{2}} \quad \text { for } \quad D=\frac{\mathcal{P}^{2}}{30}
$$

as appears in the generalised Taylor model (6);

- whereas the fourth term (the first on the second line above) is an $\mathcal{O}\left(h^{4}\right)$ estimate of the longitudinal advection at the mean velocity $\mathcal{P}$;

- the fifth term is the first contribution to the skewness of solutions as seen in the corresponding term in (6);

- and lastly, the sixth term contributes to the kurtosis.

The equivalent PDE for the low order holistic discretisation (15) is

$$
\frac{\partial c}{\partial t}=-\mathcal{P} \frac{\partial c}{\partial x}+\left(\frac{2}{3}+\frac{\mathcal{P}^{2}}{30}\right) \frac{\partial^{2} c}{\partial x^{2}}-\frac{2 \mathcal{P}}{45} \frac{\partial^{3} c}{\partial x^{3}}+\frac{2}{135} \frac{\partial^{4} c}{\partial x^{4}}+\mathcal{O}\left(h^{2}\right),
$$

The first two terms from this equivalent PDE are precisely the first two terms in the generalised Taylor model (6). The higher order terms are also components of the higher order generalised Taylor model; presumably higher order terms in the interelement coupling parameter $\gamma$ will complete the higher order terms. But see that the vitally important shear dispersion term appears as a $\gamma^{2} \mathcal{P}^{2}$ term. 
In the interesting regime of large Peclet number,

$$
\frac{\partial c_{j}}{\partial t} \approx \cdots+\left(\frac{\mathcal{P}^{2} h^{2}}{72}-\frac{\mathcal{P}^{2} h^{4}}{20}\right) h^{-4} \delta^{4} c_{j}
$$

this last term is stablising only when the grid spacing $h$ is not too small: $h^{2} \geq 5 / 18$. As is usual in coarse descriptions of shear dispersion $[14,5$, e.g.] we cannot model structures which are too small in the longitudinal direction. Even though the equivalent PDE (18) is not stable for small $h$, interestingly this holistic model is stable for finite $h$.

\section{Inlet and outlet boundary conditions are easily incorporated}

A great advantage of using this holistic technique to discretisation is that inlet and outlet boundary conditions are appropriately and easily incorporated into the analysis. Introduce a Dirichlet inlet boundary condition of prescribed concentration and the Neumann zero diffusive flux outlet boundary condition as shown in Figure 4. Here we construct discretisations to be used for the elements near the inlet and outlet.

We follow a similar treatment of the physical boundaries as introduced in [9]. We apply the Dirichlet inlet boundary condition at a grid point, say

$$
c\left(x_{0}, y, t\right)=a(t) .
$$

This may be viewed as making the first element a little longer as seen in Figure 5. We apply the Neumann outlet boundary condition at the right hand edge of the right most element, say

$$
\frac{\partial c}{\partial x}=0 \quad \text { at } \quad x=x_{m}+h / 2,
$$




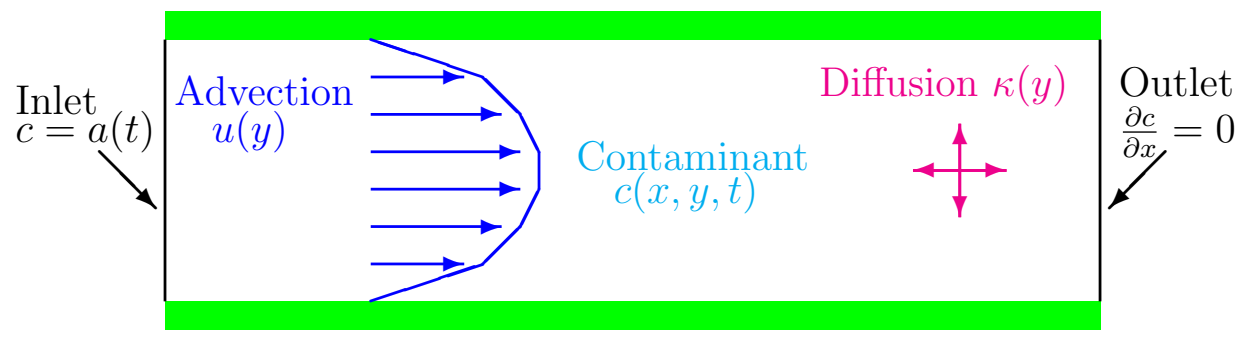

Figure 4: The fixed upstream boundary condition and the zero diffusive flux downstream boundary condition
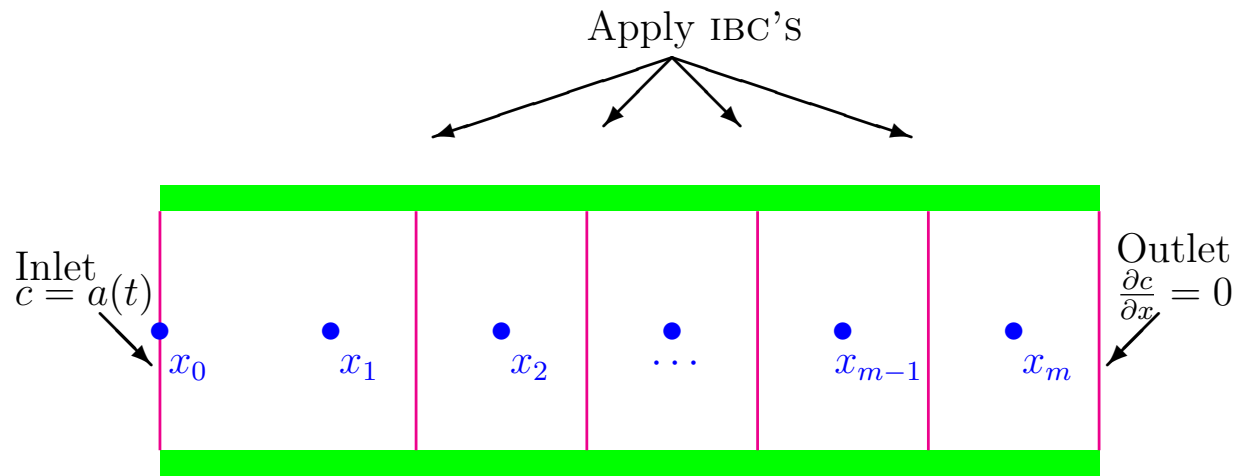

Figure 5: The physical boundary conditions are applied to the left hand side of the first element and the right hand side of the last element

as shown in Figure 5. Here we restrict attention to inlet and outlet conditions which are constant across the channel as the analysis can be done algebraically; more general inlet conditions appear to need numerical solutions for the subgrid problem.

To apply centre manifold theory, rewrite (20) in the form analogous to the IBC (7)

$$
c_{1}\left(x_{0}, y, t\right)-c_{1}\left(x_{1}, y, t\right)=\gamma\left(c_{1}\left(x_{0}, y, t\right)-a(t)\right) \quad \text { at } \quad x=x_{0},
$$

and use (21) directly. The arguments of $\S 3$ still apply to ensure 
theoretical support for the numerical model. The construction of the discretisation then proceeds as before but with special treatment of elements near the inlet or outlet. After constructing the model with $\mathcal{O}\left(\gamma^{3}, \mathcal{P}^{3}\right)$ errors and setting the coupling parameter $\gamma=1$, we find the discretisations to be used near the inlet and outlet are

$$
\begin{aligned}
\frac{\partial c_{1}}{\partial t}= & \frac{1}{18 h^{2}}\left(-c_{3}+16 c_{2}-29 c_{1}+14 a\right)+\frac{\mathcal{P}}{12 h}\left(c_{3}-8 c_{2}+7 a\right) \\
& +\frac{1}{135 h^{4}}\left(2 c_{3}-8 c_{2}+10 c_{1}-4 a\right)+\frac{\mathcal{P}}{45 h^{3}}\left(-c_{3}+2 c_{2}-a\right) \\
+ & \frac{\mathcal{P}^{2}}{80}\left(-4 c_{3}+16 c_{2}-25 c_{1}+13 a\right) \\
+ & \frac{\mathcal{P}^{2}}{360 h^{2}}\left(5 c_{3}-8 c_{2}+7 c_{1}-4 a\right)+\mathcal{O}\left(\mathcal{P}^{3}, \dot{a}\right) \\
\frac{\partial c_{2}}{\partial t}= & \frac{1}{18 h^{2}}\left(-c_{4}+16 c_{3}-30 c_{2}+16 c_{1}-a\right) \\
& +\frac{\mathcal{P}}{12 h}\left(c_{4}-8 c_{3}+8 c_{1}-a\right) \\
& +\frac{1}{135 h^{4}}\left(2 c_{4}-8 c_{3}+12 c_{2}-8 c_{1}+2 a\right) \\
& +\frac{\mathcal{P}}{45 h^{3}}\left(-c_{4}+2 c_{3}-2 c_{1}+a\right) \\
& +\frac{\mathcal{P}^{2}}{20}\left(-c_{4}+4 c_{3}-6 c_{2}+4 c_{1}-a\right) \\
& +\frac{\mathcal{P}^{2}}{360 h^{2}}\left(5 c_{4}-8 c_{3}+6 c_{2}-8 c_{1}+5 a\right)+\mathcal{O}\left(\mathcal{P}^{3}, \dot{a}\right),(24)
\end{aligned}
$$

$$
\begin{aligned}
\frac{\partial c_{m-1}}{\partial t}= & \frac{1}{18 h^{2}}\left(15 c_{m}-30 c_{m-1}+16 c_{m-2}-c_{m-3}\right) \\
& +\frac{\mathcal{P}}{288 h}\left(-167 c_{m}-c_{m-1}+192 c_{m-2}-24 c_{m-3}\right) \\
& +\frac{1}{135 h^{4}}\left(-6 c_{m}+12 c_{m-1}-8 c_{m-2}+2 c_{m-3}\right)
\end{aligned}
$$




$$
\begin{aligned}
& +\frac{\mathcal{P}}{1080 h^{3}}\left(23 c_{m}+c_{m-1}-48 c_{m-2}+24 c_{m-3}\right) \\
& +\frac{\mathcal{P}^{2}}{23040}\left(3401 c_{m}-6857 c_{m-1}+4608 c_{m-2}-1152 c_{m-3}\right) \\
& +\frac{\mathcal{P}^{2}}{17280 h^{2}}\left(-133 c_{m}+277 c_{m-1}-384 c_{m-2}+240 c_{m-3}\right) \\
& +\mathcal{O}\left(\mathcal{P}^{3}\right)
\end{aligned}
$$

$$
\begin{aligned}
\frac{\partial c_{m}}{\partial t}= & \frac{1}{18 h^{2}}\left(-14 c_{m}+15 c_{m-1}-c_{m-2}\right) \\
& +\frac{\mathcal{P}}{1440 h}\left(-937 c_{m}+1073 c_{m-1}-136 c_{m-2}\right) \\
& +\frac{1}{135 h^{4}}\left(4 c_{m}-6 c_{m-1}+2 c_{m-2}\right) \\
& +\frac{\mathcal{P}}{1080 h^{3}}\left(27 c_{m}-52 c_{m-1}+25 c_{m-2}\right) \\
& +\frac{\mathcal{P}^{2}}{2560}\left(-431 c_{m}+580 c_{m-1}-149 c_{m-2}\right) \\
& +\frac{\mathcal{P}^{2}}{17280 h^{2}}\left(208 c_{m}-461 c_{m-1}+253 c_{m-2}\right) \\
& +\mathcal{O}\left(\mathcal{P}^{3}\right) .
\end{aligned}
$$

Numerical experiments using (15) and (23-26) have shown good agreement with numerical solutions of the Taylor model, but a more complete comparison awaits further research.

\section{Conclusion}

Centre manifold theory provides a powerful new approach to deriving finite difference models of dynamical systems. This application of the holistic technique provides a direct link between the 2D 
advection-diffusion dynamics (1) and the 1D numerical model (15). The one dimensional holistic discretisation was derived from the original 2D equations without first deriving a 1D continuum model such as the Taylor model. We also found the shear dispersion term appears in the holistic discretisation in the low order approximation (15). Physical boundary conditions are also easily incorporated into the analysis.

To extend this work we must analyse boundary conditions that vary across the channel and in time. This requires numerical construction of the subgrid scale field in each element and is the subject of further research.

\section{References}

[1] J. Carr. Applications of centre manifold theory, volume 35 of Applied Math. Sci. Springer-Verlag, 1981. C515

[2] J. Carr and R.G. Muncaster. The application of centre manifold theory to amplitude expansions. II. Infinite dimensional problems. J. Diff. Eqns, 50:280-288, 1983. C515, C519, C520

[3] T. MacKenzie and A. J. Roberts. Holistic finite differences accurately model the dynamics of the Kuramoto-Sivashinsky equation. ANZIAM J., 42(E):C918-C935, 2000. [Online] http://anziamj . austms.org.au/V42/CTAC99/Mack C520

[4] T. MacKenzie and A. J. Roberts. The dynamics of reaction diffusion equations lead to a holistic discretisation. In R. L. May, G. F. Fitz-Gerald, and I. H. Grundy, editors, EMAC 2000 Proceedings. Proceedings of the fourth biennial 
Engineering Mathematics and Applications Conference, pages 199-202, 2000. C518, C520

[5] G. N. Mercer and A. J. Roberts. A complete model of shear dispersion in pipes. Jap. J. Indust. Appl. Math., 11:499-521, 1994. C524

[6] A. J. Roberts. Boundary conditions for approximate differential equations. J. Austral. Math. Soc. B, 34:54-80, 1992. C515

[7] A.J. Roberts. Low-dimensional modelling of dynamics via computer algebra. Comput. Phys. Comm., 100:215-230, 1997. C521

[8] A. J. Roberts. Holistic discretisation ensures fidelity to Burgers' equation. Applied Numerical Modelling, 37:371-396, 2001. C515, C518, C520, C521

[9] A. J. Roberts. Derive boundary conditions for holistic discretisations of burgers' equation. In Proc. of 10th Computational Techniques and Applications Conference CTAC-2001, volume 44 of ANZIAM J., 2003. [Online] http://anziamj . austms . org. au/V44/CTAC2001/Robe. C515, C520, C524

[10] A. J. Roberts. A holistic finite difference approach models linear dynamics consistently. Mathematics of Computation, 72:247-262, 2003. C518, C520

[11] S. Rosencrans. Taylor dispersion in curved channels. SIAM J. Appl. Math., 57:1216-1241, 1997. C517

[12] R. Smith. Entry and exit conditions for flow reactors $I M A$ J. Appl. Math. 41:1-20, 1988. C515 
[13] G. I. Taylor. Conditions under which dispersion of a solute in a stream of solvent can be used to measure molecular diffusion. Proc. Roy. Soc. Lond. A, 225:473-477, 1954. C514

[14] S. D. Watt and A. J. Roberts. The accurate dynamic modelling of contaminant dispersion in channels. SIAM J. Appl Math, 55(4):1016-1038, 1995. C515, C516, C524 